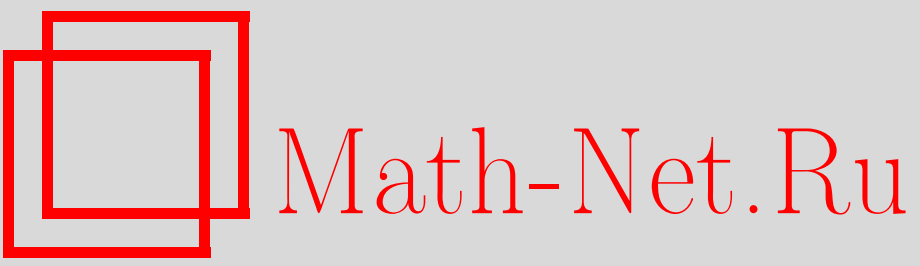

И. Э. Степанова, А. В. Щепетилов, Задача двух тел на пространствах постоянной кривизны. II. Спектральные свойства гамильтониана, ТМФ, 2000, том 124, номер 3, 481-489

DOI: https://doi.org/10.4213/tmf652

Использование Общероссийского математического портала Math-Net.Ru подразумевает, что вы прочитали и согласны с пользовательским соглашением

http://www.mathnet.ru/rus/agreement

Параметры загрузки:

IP : 3.85 .5 .30

26 апреля 2023 г., 03:11:49 
ТЕОРЕТИЧЕСКАЯ

И МАТЕМАТИЧЕСКАЯ

ФИЗИКА

Том 124, № 3

сентябрь, 2000

(C) $\mathbf{2 0 0 0}$ г.

И.Э. Степанова* ${ }^{*}$ А. В. Щепетилов ${ }^{\dagger}$

\section{ЗАДАЧА ДВУХ ТЕЛ НА ПРОСТРАНСТВАХ ПОСТОЯННОЙ КРИВИЗНЫ. ІІ. СПЕКТРАЛЬНЫЕ СВОЙСТВА ГАМИЛЬТОНИАНА}

Рассмотрена задача двух тел с центральным взаимодействием на односвязных пространствах постоянной кривизны произвольной размерности. Найденное в части I нашей работы явное выражение квантового гамильтониана через радиальный диффференциальный оператор и генераторы групп изометрий конфигурационного пространства использовано для построения его самосопряженного расширения. В случае пространства $\mathbb{S}^{3}$ для ряда потенциалов получены некоторые точные спектральные серии.

\section{1. ВВЕДЕНИЕ}

Данная работа является непосредственным продолжением работы [1]. Не повторяя результаты последней, мы используем прямые ссылки вида (I.n) на формулу (n) работы [1].

Для интегрируемости двухчастичной задачи с центральным потенциалом взаимодействия на пространствах постоянной кривизны недостаточно ее априори существуюших симметрий. В настоящее время для нее неизвестны какие-либо скрытые симметрии. В работах $[2,3]$ найдены потенциалы на пространствах $\mathbb{S}^{n}$ и $\mathbb{H}^{n}$, для которых одночастичный гамильтониан допускает разделение переменных более чем в одной системе координат. Однако для двухчастичной задачи с центральным взаимодействием неизвестны потенциалы, допускаюшие разделение переменных хотя бы в одной системе координат. В работе [4] гамильтониан двухчастичной задачи при $n=2$ был выражен через радиальный дифференциальный оператор и генераторы групп изометрий. С помощью теории представлений для групा $\mathrm{SO}(3)$ и $\mathrm{SO}(1,2)$ задача на собственные значения гамильтониана была сведена к последовательности систем (конечных в случае пространства $\mathbb{S}^{2}$ ) обыкновенных дифференциальных уравнений второго порядка, нумеруемых неприводимыми представлениями данных групп. При равенстве масс частищ в пространстве $\mathbb{S}^{2}$ возможно дальнейшее упрошение задачи, так как из указанной последовательности систем обыкновенных дифференциальных уравнений выделяются восемь отдельных уравнений, из которых для некоторых потенциалов взаимодействия можно

* Объединенный институт физики Земли РАН, Москва, Россия

${ }^{\dagger}$ Московский государственный университет, Москва, Россия. E-mail: alexey@quant.phys.msu.su 
получить явные формулы для спектральных серий. В работе [1] классическая и квантовая задачи были рассмотрены совместно и, в частности, получены выражения для квантовых гамильтонианов через радиальный дифференциальный оператор и генераторы групп изометрий $\mathrm{SO}(n+1), \mathrm{SO}(1, n)$ для произвольных $n$. В настоящей работе мы используем полученные в [1] выражения при доказательстве самосопряженности двухчастичных гамильтонианов, для случая пространства $\mathbb{S}^{3}$ выделяем все отдельные дифференциальные уравнения на спектральные значения и для ряда потенциалов взаимодействия получаем явный вид спектральных серий, соответствующих этим дифференциальным уравнениям. Тем самым, хотя двухчастичная задача на пространствах $\mathbb{S}^{n}$ и $\mathbb{H}^{n}$, по-видимому, не является интегрируемой в каком-либо смысле, некоторые ее спектральные значения могут быть получены явно. Такая ситуация характеризует квазиточнорешаемые модели [5]. Однако имеется два сушественных отличия. Во-первых, обычно квазиточнорешаемые модели являются искусственно построенными. Во-вторых, точные спектральные значения, как правило, выделяются из одного дифференциального уравнения со специально подобранным потенциалом, а в данной задаче мы выделяем отдельные дифференциальные уравнения из систем обыкновенных дифференциальных уравнений.

\section{2. САМОСОПРЯЖЕННОСТЬ ДВУХЧАСТИЧНЫХ ГАМИЛЬТОНИАНОВ}

В евклидовом пространстве самосопряженность многочастичных гамильтонианов с парным взаимодействием доказывается с помощью галилеевой инвариантности, аналог которой отсутствует для пространств $\mathbb{S}^{n}$ и $\mathbb{H}^{n}[6]$. Самосопряженность одночастичных гамильтонианов с сингулярными потенциалами, не ограниченными снизу, эффективнее всего устанавливается при помоши теории возмущений соответствующих им квадратичных форм. Ключевым моментом доказательства для конкретных гамильтонианов является оценка, названная в [6] “принципом неопределенности”, следующего вида:

$$
(U \psi, \psi) \leqslant\|\nabla \psi\|^{2},
$$

где $(\cdot, \cdot)$ - скалярное произведение в $\mathcal{L}^{2}\left(\mathbb{R}^{3}\right), \nabla$ - оператор градиента, а $U$ - потенциал. Получение и использование подобной оценки в пространствах $\mathbb{S}^{n}$ и $\mathbb{H}^{n}$ потребует, как будет показано ниже, некоторых модификаций доказательства. Мы не будем стремиться к максимальной общности, имея в виду, прежде всего, физически осмысленные потенциалы.

Операторы Лапласа-Бельтрами на пространствах $\mathbb{S}^{n}$ и $\mathbb{H}^{n}$ самосопряжены на областях

$$
\begin{aligned}
& D_{s}(\Delta)=\left\{\phi \in \mathcal{L}^{2}\left(\mathbb{S}^{n}\right) \mid \Delta \phi \in \mathcal{L}\left(\mathbb{S}^{n}\right)\right\} \\
& D_{h}(\Delta)=\left\{\phi \in \mathcal{L}^{2}\left(\mathbb{H}^{n}\right) \mid \Delta \phi \in \mathcal{L}\left(\mathbb{H}^{n}\right)\right\}
\end{aligned}
$$

и самосопряжены в сушественном на подпространствах этих пространств, состоящих из гладких функций с компактным носителем. Отсюда следует самосопряженность свободных двухчастичных гамильтонианов на соответствуюших тензорных произведениях 
данных функциональных пространств. Опираясь на этот факт, по теории возмушений квадратичных форм мы получим самосопряженность двухчастичных гамильтонианов с взаимодействием.

Пусть $(\cdot, \cdot)$ - скалярное произведение на слоях кокасательного расслоения $Q_{s}$, отвечающее метрике $\tilde{g}_{s},\|\cdot\|$ - соответствуюшая норма, $\nabla$ - соответствуюший оператор градиента, а $f, \psi \in C^{\infty}\left(Q_{s}\right)$ - вешественные функции ${ }^{1)}$, причем $f$ постоянна на слоях $F_{r}^{s}$, т.е. $f=f(r)$. Тогда

$$
\begin{aligned}
\|\nabla \psi\|^{2} & =\left\|\frac{\nabla(f \psi)}{f}-\frac{\psi \nabla f}{f}\right\|^{2} \geqslant \frac{\psi^{2}\|\nabla f\|^{2}}{f^{2}}-\frac{2 \psi(\nabla f, \nabla(f \psi))}{f^{2}}= \\
& =\frac{\psi^{2} \tilde{g}_{s}^{r r}}{f^{2}}\left(f^{\prime}\right)^{2}-\frac{2 \psi}{f^{2}} \tilde{g}_{s}^{r r} f^{\prime} \frac{\partial}{\partial r}(f \psi) .
\end{aligned}
$$

Из (І.12) вытекает, что $\tilde{g}^{r r}=\left(1+r^{2}\right)^{2} /\left(8 R^{2} m\right)$. Интегрируя по $Q_{s}$ с мерой $d \mu_{s}$, получаем

$$
\begin{aligned}
\int_{Q_{s}}\|\nabla \psi\|^{2} d \mu_{s} \geqslant & \frac{1}{8 R^{2} m} \int_{Q_{s}}\left[\frac{\left(f^{\prime}\right)^{2} \psi^{2}}{f^{2}}-\frac{2 \psi f^{\prime}}{f^{2}} \frac{\partial}{\partial r}(f \psi)\right]\left(1+r^{2}\right)^{2} d \mu_{s}= \\
= & \frac{1}{8 R^{2} m} \int_{Q_{s}} \frac{\left(f^{\prime}\right)^{2} \psi^{2}}{f^{2}}\left(1+r^{2}\right)^{2} d \mu_{s}- \\
& -\frac{1}{4 R^{2} m} \int_{F_{r}^{s}} \int_{0}^{\infty} \frac{\psi f^{\prime} r^{n-1}}{f^{2}\left(1+r^{2}\right)^{n-2}} \frac{\partial}{\partial r}(f \psi) d r d \mu_{f} .
\end{aligned}
$$

Попробуем подобрать функцию $f$ так, чтобы для любых $\psi$ функция

$$
\frac{\psi f^{\prime} r^{n-1}}{f^{2}\left(1+r^{2}\right)^{n-2}} \frac{\partial}{\partial r}(f \psi)
$$

имела вид $(\partial / \partial r)\left(\phi(r) \psi^{2}\right)$. При этом возможно тождественное обрашение последнего интеграла в нуль. Решая систему уравнений

получаем

$$
\frac{\left(f^{\prime}\right)^{2} r^{n-1}}{f^{2}\left(1+r^{2}\right)^{n-2}}=\phi^{\prime}, \quad \frac{f^{\prime} r^{n-1}}{f\left(1+r^{2}\right)^{n-2}}=2 \phi,
$$

$$
\begin{aligned}
& \phi(r)=-\left[4 \int \frac{\left(1+r^{2}\right)^{n-2}}{r^{n-1}} d r\right]^{-1},
\end{aligned}
$$

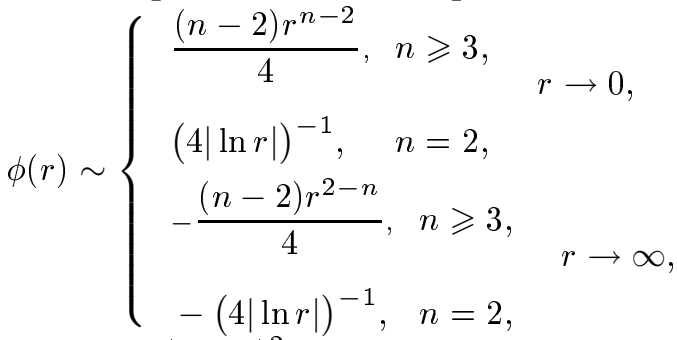

$$
\begin{aligned}
& \left(\frac{f^{\prime}}{f}\right)^{2}(r) \sim\left\{\begin{array}{ll}
\frac{(n-2)^{2}}{4 r^{2}}, & n \geqslant 3, \\
\left(4 r^{2} \ln ^{2} r\right)^{-1}, & n=2,
\end{array} \quad r \rightarrow 0, \quad r \rightarrow \infty .\right.
\end{aligned}
$$

\footnotetext{
1) Все функциональные пространства предполагаются состоящими из комплекснозначных функций.
} 
Нетрудно убедиться, что при любом выборе постоянной интегрирования для функции $\phi(r)$ она терпит разрыв в некоторой точке, чего не происходит, например, в евклидовом случае при $n \geqslant 3$. Пусть $\omega_{\delta}:=\left\{x \in Q_{s} \mid r(x)<\delta\right\}$, а $\omega_{\delta}^{\prime}:=\left\{x \in Q_{s} \mid r(x)>\delta^{-1}\right\}$. Выберем $\delta>0$ так, что функция $\phi(r)$ непрерывна на множестве $\left(\omega_{2 \delta} \cup \omega_{2 \delta}^{\prime}\right) \backslash\left(F_{0}^{s} \cup F_{\infty}^{s}\right)$. На пространстве $Q_{s} \backslash\left(F_{0}^{s} \cup F_{\infty}^{s}\right)$ функция $f^{\prime} / f$ непрерывна там же, где и функция $\phi(r)$, и, как и $\phi(r)$, не обрашается в нуль при $r \neq 0, \infty$. Положим

$$
u_{n}(r)= \begin{cases}r^{-2}+r^{2}, & n \geqslant 3, \\ \frac{r^{-2}+r^{2}}{\ln ^{2} r}, & n=2,\end{cases}
$$

и выберем постоянную $\kappa>0$ так, чтобы выполнялось неравенство

$$
\kappa u_{n}(r) \leqslant \frac{\left(1+r^{2}\right)^{2}\left(f^{\prime}\right)^{2}}{8 R^{2} m f^{2}}
$$

Тогда из (1) и (2) получаем для функции $\psi \in C^{\infty}\left(Q_{s}\right), \operatorname{supp} \psi \subset \omega_{\delta} \cup \omega_{\delta}^{\prime}$,

$$
\kappa \int_{Q_{s}} u_{n}(r)|\psi|^{2} d \mu_{s} \leqslant \int_{Q_{s}}\|\nabla \psi\|^{2} d \mu_{s}
$$

Записывая неравенство (3) отдельно для вешественной и мнимой частей комплекснозначной функции, мы приходим к справедливости оценки (3) для произвольной функции $\psi \in C^{\infty}\left(Q_{s}\right)$ с носителем в $\omega_{\delta} \cup \omega_{\delta}^{\prime}$.

ТЕОрема 1. Пусть гладкая на пространстве $Q_{s} \backslash\left(F_{0}^{s} \cup F_{\infty}^{s}\right)$ вещественная функиия $U=o\left(u_{n}(r)\right)$ при $r \rightarrow 0, \infty$ равномерно по координатам на слоях $F_{r}^{s}$. Тогда двухчастичный гамильтониан $\widehat{H}_{s}$ самосопряжсен в существенном на любой существенной области самосопряженности свободного гамильтониана $\widehat{H}_{0}^{s} . \quad$ В частности, $C^{\infty}\left(Q_{s}\right)$ есть область самосопря⿻сенности в существенном для $\widehat{H}_{s}$.

ДокАЗАТЕЛЬСТВо. В соответствии с теоремой Х.17 книги [6] достаточно доказать, что $\forall \psi \in C^{\infty}\left(Q_{s}\right)$ справедливо неравенство

$$
\int_{Q_{s}}|U||\psi|^{2} d \mu_{s} \leqslant a \int_{Q_{s}} \bar{\psi} \widehat{H}_{0}^{s} \psi d \mu_{s}+b \int_{Q_{s}}|\psi|^{2} d \mu_{s}
$$

где $0<a<1, \quad b \in \mathbb{R}$. Зафиксируем произвольное $a \in(0,1)$. Выберем функции $\chi \in C^{\infty}\left(Q_{s}\right)$ так, чтобы $\operatorname{supp} \chi \subset \omega_{\delta} \cup \omega_{\delta}^{\prime},\left.\chi\right|_{\omega_{\delta / 2} \cup \omega_{\delta / 2}^{\prime}} \equiv 1,0 \leqslant \chi \leqslant 1$. Пусть теперь $0<\varepsilon \leqslant \delta / 2$, причем $|U(x)| \leqslant a \kappa u_{n}(r(x)) / 2, x \in \omega_{\varepsilon} \cup \omega_{\varepsilon}^{\prime}$. Пусть

$$
c:=\sup _{x \in Q_{s} \backslash\left(\omega_{\varepsilon} \cup \omega_{\varepsilon}^{\prime}\right)}|U(x)|
$$

а $\psi \in C^{\infty}\left(Q_{s}\right)$. Тогда

$$
\int_{Q_{s}}|U||\psi|^{2} d \mu_{s} \leqslant \frac{a}{2} \kappa \int_{\omega_{\varepsilon} \cup \omega_{\varepsilon}^{\prime}} u_{n}(x)|\chi \psi|^{2} d \mu_{s}+c \int_{Q_{s}}|\psi|^{2} d \mu_{s} .
$$


K первому интегралу можно применить оценку (3), что дает

$$
\kappa \int_{\omega_{\varepsilon} \cup \omega_{\varepsilon}^{\prime}} u_{n}(x)|\chi \psi|^{2} d \mu_{s} \leqslant \int_{Q_{s}}\|\nabla(\chi \psi)\|^{2} d \mu_{s} \leqslant 2 \int_{Q_{s}}\left(|\nabla \psi|^{2}+|\nabla \chi|^{2}|\psi|^{2}\right) d \mu_{s},
$$

откуда получаем

$\int_{Q_{s}}|U||\psi|^{2} d \mu_{s} \leqslant a \int_{Q_{s}}\|\nabla \psi\|^{2} d \mu_{s}+b \int_{Q_{s}}|\psi|^{2} d \mu_{s}=a \int_{Q_{s}} \bar{\psi} \widehat{H}_{0}^{s} \psi d \mu_{s}+b \int_{Q_{s}}|\psi|^{2} d \mu_{s}$ где $b=c+2 \sup _{Q_{s}}\left(|\nabla \chi|^{2}\right)$.

Для пространства $\mathbb{H}^{n}$ справедлив аналогичный результат.

ТЕОрема 2. Пусть гладкая на $Q_{h} \backslash F_{0}^{h}$ и ограниченная на любом множестве вида $W \subset \subset Q_{h} \backslash F_{0}^{h}$ вещественная функция $U=o\left(r^{-2}\right)$ при $r \rightarrow 0$ равномерно по координатам на слоях $F_{r}^{h}$. Тогда двухчастичный гамильтониан $\widehat{H}_{h}$ самосопряжен в существенном на любой существенной области самосопряхсенности свободного гамильтониана $\widehat{H}_{0}^{h}$. В частности, $C^{\infty}\left(Q_{h}\right)$ есть область самосопряжсенооти в существенном для $\widehat{H}_{h}$.

\section{CПEKTP OПEPATOPA $\widehat{H}_{s}$}

Разложив пространство $\mathcal{L}^{2}\left(\mathrm{SO}(n+1), K, d \eta_{s}\right)$ в прямую сумму пространств конечномерных неприводимых представлений $T_{m}$ группы $\mathrm{SO}(n+1)$ (как это было сделано в работе [4]), мы получим выражение для ограничения оператора $\widehat{H}_{s}$ на $\mathcal{L}^{2}\left(\mathbb{R}_{+}, d \nu_{s}\right) \otimes T_{m}$ в виде матричного обыкновенного дифференциального оператора.

В этой связи необходимо определить, какие неприводимые представления группы $\mathrm{SO}(n+1)$ содержатся в пространстве $\mathcal{L}^{2}\left(\mathrm{SO}(n+1), K, d \eta_{s}\right)$. Известно (см., например, [7]), что все неприводимые представления компактной группы Г содержатся в ее регулярном представлении в пространстве $\mathcal{L}^{2}(\Gamma, d \eta)$. Задача о характеризации тех неприводимых представлений группы $\mathrm{SO}(n)$, которые содержатся в ее представлении в пространстве $\mathcal{L}^{2}(\mathrm{SO}(n) / \mathrm{SO}(n-m))$, была решена в работах [8]. Каждое неприводимое представление группы $\mathrm{SO}(n)$ однозначно характеризуется своим старшим весом [9]: $m=\left(m_{1}, m_{2}, \ldots, m_{k}\right)$, где $k=n / 2$ для четных $n$ и $k=(n-1) / 2$ для нечетных $n$. Как было показано в работах [8], в пространство $\mathcal{L}^{2}(\mathrm{SO}(n) / \mathrm{SO}(n-m))$ входят только те неприводимые представления групшы $\mathrm{SO}(n)$, для которых лишш первые $m$ компонент старшего веса могут быть отличны от нуля. Таким образом, в пространстве $\mathcal{L}^{2}\left(\mathrm{SO}(n+1), K, d \eta_{s}\right)$ содержатся все неприводимые представления групшы $\mathrm{SO}(n+1)$ при $n=2,3,4$.

В работе [10] получены явные (и весьма громоздкие) выражения для действия инфинитезимальных генераторов группы $\mathrm{SO}(n)$ на базисные элементы ее неприводимого представления, характеризуемые схемами Гельфанда-Цетлина.

Таким образом, матричный обыкновенный дифференциальный оператор, соответствующий ограничению $\widehat{H}_{s}$ на $\mathcal{L}^{2}\left(\mathbb{R}_{+}, d \nu_{s}\right) \otimes T_{m}$, может быть вычислен явно. Интересно найти такие одномерные подпространства $T_{m}^{\prime}$ пространств $T_{m}$, что пространства 
$\mathcal{L}^{2}\left(\mathbb{R}_{+}, d \nu_{s}\right) \otimes T_{m}^{\prime}$ инвариантны относительно оператора $\widehat{H}_{s}$, так как в этом случае можно выделить отдельные уравнения на собственные значения двухчастичного гамильтониана на пространстве $\mathbb{S}^{n}$, которые для некоторых потенциалов $U(r)$ могут быть явно решены. Из формул (I.11) и (I.13) видно, что при $m_{1} \neq m_{2}$ для этого необходимо и достаточно найти общие собственные функции из пространства $T_{m}$ для операторов

$$
D_{1}=\left(Y_{1}^{s, l}\right)^{2}, \quad D_{2}=\sum_{i=2}^{n}\left(X_{1 i}^{s, l}\right)^{2}, \quad D_{3}=\sum_{i=2}^{n}\left(Y_{i}^{s, l}\right)^{2}, \quad D_{4}=\sum_{i=2}^{n}\left\{X_{1 i}^{s, l}, Y_{i}^{s, l}\right\},
$$

а при $m_{1}=m_{2}$ в силу условия $B_{s}(r) \equiv 0$ - общие собственные функции операторов $D_{1}, D_{2}$ и $D_{3}$.

Ввиду отсутствия метода нахождения общих собственных векторов некоммутативных операторов, отличного от явных вычислений, мы ограничимся рассмотрением практически важного случая сферы $\mathbb{S}^{3}$. Напомним, что случай сферы $\mathbb{S}^{2}$ был рассмотрен в работе [4].

Вследствие изоморфизмов $\mathrm{SO}(4) \simeq \mathrm{SU}(2) \times \mathrm{SU}(2) /\{ \pm(1 \otimes 1)\}, \mathrm{SO}(3) \simeq \mathrm{SU}(2) /\{ \pm 1\}$ неприводимые представления группы $\mathrm{SO}(4)$ являются тензорными произведениями представлений группы SU(2), отвечающих одновременно целым или полуцелым значениям веса.

Базис алгебры so(4), состоящий из элементов $L_{1}, L_{2}, L_{3}, G_{1}, G_{2}, G_{3}$, определяемых равенствами

$$
\begin{aligned}
L_{1} & =\frac{1}{2}\left(X_{23}^{s}+Y_{1}^{s}\right), & L_{2} & =\frac{1}{2}\left(X_{31}^{s}+Y_{2}^{s}\right), \\
G_{1} & =\frac{1}{2}\left(X_{23}^{s}-Y_{1}^{s}\right), & L_{3} & =\frac{1}{2}\left(X_{12}^{s}+Y_{3}^{s}\right), \\
& =\frac{1}{2}\left(X_{31}^{s}-Y_{2}^{s}\right), & G_{3} & =\frac{1}{2}\left(X_{12}^{s}-Y_{3}^{s}\right),
\end{aligned}
$$

соответствует разложению $\mathrm{so}(4)=\mathrm{so}(3) \oplus \mathrm{so}(3)$. При этом имеют место коммутационные соотношения

$$
\left[L_{m}, L_{j}\right]=\sum_{k=1}^{3} \varepsilon_{m j k} L_{k}, \quad\left[G_{m}, G_{j}\right]=\sum_{k=1}^{3} \varepsilon_{m j k} G_{k}, \quad\left[L_{m}, G_{j}\right]=0, \quad m, j=1,2,3
$$

где $\varepsilon_{m j k}-$ полностью антисимметричный тензор, $\varepsilon_{123}=1$. Неприводимые представления группы $\mathrm{SO}(4)$ нумеруются тем самым двумя одновременно целыми или полуцелыми неотрицательными числами $l_{1}$ и $l_{2}$, и в пространстве неприводимого представления можно выбрать базис, элементы которого $\left|n_{1}, n_{2}\right|=\left|n_{1}\right| \otimes\left|n_{2}\right|$ нумеруются числами $n_{1}$ и $n_{2}$, целыми или полуцелыми одновременно с $l_{1}$ и $l_{2}$, причем $-l_{j} \leqslant n_{j} \leqslant l_{j}, j=1,2$, и выполняются следующие соотношения:

$$
\begin{aligned}
L_{1}\left|n_{1}\right| & =-i n_{1}\left|n_{1}\right|, \quad G_{1}\left|n_{2}\right|=-i n_{2}\left|n_{2}\right|, \\
H_{+}\left|n_{1}\right| & =-\sqrt{\left(l_{1}-n_{1}\right)\left(l_{1}+n_{1}+1\right)}\left|n_{1}+1\right|, \\
H_{-}\left|n_{1}\right| & =-\sqrt{\left(l_{1}+n_{1}\right)\left(l_{1}-n_{1}+1\right)}\left|n_{1}-1\right|, \\
P_{+}\left|n_{2}\right| & =-\sqrt{\left(l_{2}-n_{2}\right)\left(l_{2}+n_{2}+1\right)}\left|n_{2}+1\right|, \\
P_{-}\left|n_{2}\right| & =-\sqrt{\left(l_{2}+n_{2}\right)\left(l_{2}-n_{2}+1\right)}\left|n_{2}-1\right|,
\end{aligned}
$$


где $H_{ \pm}:=i L_{2} \pm L_{3}, P_{ \pm}:=i G_{2} \pm G_{3}$. Операторы $D_{m}, m=1,2,3$, имеют вид

$$
\begin{gathered}
D_{1}=L_{1}^{2}+G_{1}^{2}-2 L_{1} G_{1}, \quad D_{2}=F_{1}+F_{2}-L_{1}^{2}-G_{1}^{2}+2 L_{2} G_{2}+2 L_{3} G_{3}, \\
D_{3}=F_{1}+F_{2}-L_{1}^{2}-G_{1}^{2}-2 L_{2} G_{2}-2 L_{3} G_{3}, \quad D_{4}=4\left(L_{2} G_{3}-L_{3} G_{2}\right),
\end{gathered}
$$

где $F_{1}:=L_{1}^{2}+L_{2}^{2}+L_{3}^{2}, F_{2}:=G_{1}^{2}+G_{2}^{2}+G_{3}^{2}$ - операторы Казимира алгебры so(3), ограничения которых на неприводимые представления являются скалярными операторами $-l_{1}\left(l_{1}+1\right)$ и $-l_{2}\left(l_{2}+1\right)$, соответственно. Следовательно, обшие собственные векторы операторов $D_{1}, D_{2}$ и $D_{3}$ должны быть собственными векторами операторов $L_{1}^{2}+G_{1}^{2}$, $L_{1} G_{1}, L_{2} G_{2}+L_{3} G_{3}$. Прямые, но громоздкие вычисления приводят к следующим пяти частично перекрываюшимся сериям обших собственных векторов операторов $D_{1}, D_{2}$ и $D_{3}$ :

1) $l_{1}=l_{2}=: l \geqslant 1 ;|l-2, l|-|l, l-2|,|-l+2,-l|-|-l,-l+2|$;

2) $l_{2} \geqslant l_{1}=1$ или $l_{1} \geqslant l_{2}=1 ;|1,-1|-|-1,1|$;

3) $l_{1}=l_{2}=: l \geqslant 1 / 2 ;|l-1, l| \pm|l, l-1|,|-l+1,-l| \pm|-l,-l+1|$;

4) $l_{2} \geqslant l_{1}=1 / 2$ или $l_{1} \geqslant l_{2}=1 / 2 ;|1 / 2,-1 / 2| \pm|-1 / 2,1 / 2|$;

5) $\left|l_{1}, l_{2}\right| ;\left|-l_{1},-l_{2}\right|$.

Только пятая серия состоит из собственных векторов оператора $D_{4}$. Разыскивая собственную функцию оператора $\widehat{H}_{s}$ в виде $\psi(r) \tilde{\psi}$, где $\tilde{\psi}-$ один из векторов серий $1-5$, мы получаем следующие уравнения на функцию $\psi(r)$ при $m_{1}=m_{2}=2 m$ в случаях $1-4$ и при произвольных $m_{1}$ и $m_{2}$ в случае 5 :

$$
\begin{gathered}
L \psi+\left(\frac{1}{2 m R^{2}}\left((3 l-2) \frac{\left(1+r^{2}\right)^{2}}{2 r^{2}}+1\right)+U-E\right) \psi=0, \quad l \geqslant 1, \\
L \psi+\left(\frac{1}{2 m R^{2}}\left(\left(l_{2}+1\right) l_{2} \frac{\left(1+r^{2}\right)^{2}}{4 r^{2}}+1\right)+U-E\right) \psi=0, \quad l_{2} \geqslant 1, \\
L \psi+\left(\frac{1}{8 m R^{2}}\left((4 l-1) \frac{\left(1+r^{2}\right)^{2}}{r^{2}}+1 \pm 2 l \frac{1-r^{4}}{r^{2}}\right)+U-E\right) \psi=0, \quad l \geqslant \frac{1}{2}, \\
L \psi+\left(\frac{1}{8 m R^{2}}\left(\left(l_{2}\left(l_{2}+1\right)+\frac{1}{4}\right) \frac{\left(1+r^{2}\right)^{2}}{r^{2}}+1 \pm\left(l_{2}+\frac{1}{2}\right) \frac{1-r^{4}}{r^{2}}\right)\right) \psi+ \\
+(U-E) \psi=0, \quad l_{2} \geqslant \frac{1}{2}, \quad\left(\frac{\left(l_{1}-l_{2}\right)^{2}}{2\left(m_{1}+m_{2}\right) R^{2}}+\frac{\left(l_{1}+l_{2}\right)\left(1+r^{2}\right)^{2}}{8 m R^{2} r^{2}}+U-E\right) \psi=0, \quad l_{1}, l_{2} \geqslant 0, \\
L \psi+(
\end{gathered}
$$

где

$$
L=-\frac{\left(1+r^{2}\right)^{3}}{8 m R^{2} r^{2}} \frac{\partial}{\partial r}\left(\frac{r^{2}}{1+r^{2}} \frac{\partial}{\partial r}\right) .
$$

Отметим, что уравнение для радиальной компоненты $\psi(r)$ собственной функции одной частицы имеет вид

$$
L \psi+\left(\frac{l(l+1)}{8 m R^{2}}\left(r^{-2}+2+r^{2}\right)+U-E\right) \psi=0, \quad l=0,1,2, \ldots
$$


Таким образом, энергетические уровни вычисляются точно из уравнений (4), (5), (8) всегда, когда они вычисляются точно для того же потенциала в одночастичном случае.

Как правило, возможность точного вычисления спектра обыкновенного дифференциального оператора обусловлена либо разрешимостью соответствующего уравнения в элементарных функциях, либо его сводимостью к гипергеометрическому уравнению (или его предельным случаям). Гипергеометрическое уравнение является, в свою очередь, частным случаем уравнения Римана, а последнее сводится к первому степенным преобразованием. Уравнение

$$
L \psi+\left(\eta r^{-2}+\nu r^{2}-E\right) \psi=0, \quad \eta, \nu=\mathrm{const}
$$

является уравнением Римана относительно переменной $\xi=r^{2}$. При $\eta, \nu>0$ соответствуюший дифференциальный оператор допускает самосопряженное расширение по Фридрихсу [4], а уровни энергии имеют вид

$$
\begin{aligned}
E_{k}= & \mu\left[k(k+1)-\frac{5}{8}+(2 k+1)\left(\sqrt{\frac{1}{16}+\frac{\eta}{\mu}}+\sqrt{\frac{1}{16}+\frac{\nu}{\mu}}\right)+\right. \\
& \left.+2 \sqrt{\frac{1}{16}+\frac{\eta}{\mu}} \sqrt{\frac{1}{16}+\frac{\nu}{\mu}}\right], \quad \mu=\frac{1}{2 m R^{2}} .
\end{aligned}
$$

Для потенциала $U=a r^{-2}+b r^{2}, a, b \geqslant 0$, из этой формулы заменой коэффициентов можно получить энергетические уровни для уравнений (4)-(8).

На сфере $\mathbb{S}^{n}$ известны аналоги кулоновского и осцилляторного потенциалов [11]

$$
U_{\mathrm{q}}=\frac{\kappa}{2 R}\left(r-\frac{1}{r}\right), \quad U_{\mathrm{o}}=\frac{2 \omega^{2} R^{2} r^{2}}{\left(1-r^{2}\right)^{2}} .
$$

Они характеризуются условием замкнутости всех траекторий классического одночастичного движения. Уравнение (9) для этих потенциалов сводится к уравнению Римана относительно переменных $u=\left(1-r^{2}\right) / r$ и $v=u^{2}$, соответственно. Однако коэффициенты уравнения (10) рациональны относительно переменных $u$ и $v$ лишь при $\eta=\nu$. Таким

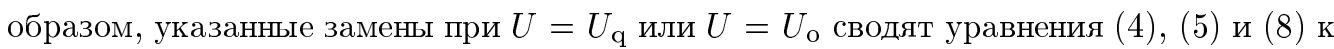
уравнению Римана и не сводят к нему уравнения (6) и (7).

Теорема 1 дает самосопряженность оператора $\widehat{H}_{s}$ при $U=U_{\mathrm{q}}$ для любых $n \geqslant 2$, а

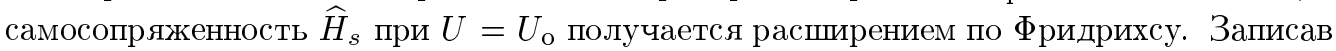
энергетические уровни для уравнения (9) при $U=U_{\mathrm{q}}$ :

$$
E_{k}=-\frac{1}{2 m R^{2}}+\frac{(k+l)^{2}}{2 m R^{2}}-\frac{m \kappa^{2}}{2(k+l)^{2}}, \quad k=1,2,3, \ldots
$$

и при $U=U_{\mathrm{o}}$ :

$E_{k}=-\frac{1}{2 m R^{2}}\left(\frac{3}{4}-\left(2 k+l+\frac{3}{2}\right)^{2}\right)+\frac{\omega\left(2 k+l+\frac{3}{2}\right)}{\sqrt{m}} \sqrt{1+\frac{1}{4 \omega^{2} R^{4} m}}, \quad k=0,1,2, \ldots$,

и сделав замену коэффициентов, можно легко получить энергетические уровни для уравнений (4), (5) и (8). 


\section{4. ЗАКЛЮЧЕНИЕ}

В данной работе мы показали, что для ряда потенциалов взаимодействия некоторые серии энергетических уровней квантовой задачи двух частиц на сфере $\mathbb{S}^{3}$ могут быть явно вычислены. Случай равных масс частиц предоставляет для этого больше возможностей.

Такое квазиточнорешаемое поведение данной задачи, по-видимому, связано с наличием некоторого множества замкнутых траекторий для соответствуюшей классической механической системы. Ясно, что при равных массах нетрудно найти замкнутые траектории в виде "окружностей", когда частицы все время остаются на одинаковом максимальном расстоянии друг от друга. Связь наличия некоторого множества замкнутых траекторий классической задачи в неинтегрируемом случае со свойствами спектра соответствуюшей квантовой задачи изучалась в ряде работ для различных ситуаций (см. [12] и приведенную там литературу). Было бы интересно найти такую связь с классической системой в явном виде в рассмотренной задаче, а также обобшить метод нахождения точных спектральных серий на случай произвольной размерности пространства $\mathbb{S}^{n}$.

Как было отмечено в работе [4], для пространства $\mathbb{H}^{2}$ оператор двухчастичной квантовой задачи, по-видимому, не имеет дискретного спектра. Вероятно, то же самое справедливо и для пространства $\mathbb{H}^{n}$.

\section{Список литературы}

[1] A. В. Щепетилов. ТМФ. 2000. Т. 124. № 2. С. 249.

[2] E. G. Kalnins, W. Miller Jr., G.S. Pogosyan. J. Math. Phys. 1996. V. 37. P. 6439; 1997. V. 38. P. 5416.

[3] E. G. Kalnins, W. Miller Jr., Ye.M. Hakobyan, G.S. Pogosyan. J. Math. Phys. 1999. V. 40. P. 2291.

[4] А.В. Щепетилов. ТМФ. 1999. Т. 118. С. 248.

[5] А.Г. Уиверидзе. ЭЧАЯ. 1989. Т. 20. С. 1185; 1992. Т. 23. С. 58.

[6] М. Рид, Б. Саймон. Методы современной математической физики. Т. 2. Гармонический анализ. Самосопряженность. М.: Мир, 1978.

[7] Н.Я. Виленкин. Специальные функции и теория представлений групп. М.: Наука, 1991.

[8] D. A. Levin. Trans. Am. Math. Soc. 1969. V. 144. P. 493; S. S. Gelbart. Trans. Am. Math. Soc. 1974. V. 192. P. 29; R. S. Strichartz. Canad. J. Math. 1975. V. 27. P. 294.

[9] А. Барут, Р. Рончка. Теория представлений групп и ее приложения. М.: Мир, 1980.

[10] U. Ottoson. Commun. Math. Phys. 1968. V. 8. P. 228; 1968. V. 10. P. 114.

[11] P. W. Higgs. J. Phys. A. 1979. V. 12. P. 309.

[12] M. C. Gutzwiller. Chaos in classical and quantum mechanics. New York: Springer, 1992. 